\title{
Survival after resection for primary lung cancer
}

\section{T Treasure, M Utley}

\section{To extend the role of surgery in lung cancer we will need an evidence base of which good observational data is a crucial component}

$\mathrm{N}$ early all that we believe in surgery and nearly all that we practise is based on observational data, usually in the form of collected series. ${ }^{1}$ Lung cancer surgery is no exception, and we rely-and will continue to rely-on the analysis of observational data. Observational research has tended to be denigrated and neglected. ${ }^{2}$ There is currently a lack of broad based data of sufficiently high quality, ${ }^{3}$ so we welcome the Norwegian population based study reported by Strand et al in this issue of Thorax. ${ }^{4}$

The Scandinavian nations have been a great resource of high quality observational data and, in the BMJ alone, we have seen many community based, population based, ${ }^{6-8}$ and registry studies, ${ }^{8}$ of which those cited ${ }^{5-8}$ are a tiny but representative sample of the many submitted. Indeed, UK groups have been attracted to these databases for their own studies. $^{59}$ Scandinavian society has a number of features that seem, to outsiders at least, to provide a fertile environment for such studies. They have well developed healthcare systems with uniformly high standards and equitable access. Also, the populations are relatively stable-for example, only 13 of $3211(0.4 \%)$ operated patients in the Norwegian study were lost due to emigration. Importantly, collection and analysis of data to inform policy is accepted in these countries as a right and proper function of the state. Strand et al relied on a population based cancer registry with obligatory entry by law, with no need for the patient's consent, and which is automatically linked to the Norwegian Central Bureau of Statistics.

Before discussing the results presented by Strand et al, it is important to make a distinction between a registry and a research database. A registry should account for all patients, albeit with a limited data set, whereas a research database generally requires much more information, albeit on a limited sample. The aphorism "some data on all of the patients versus all of the data on some of the patients" sums it up. The Norwegian report is from a registry; its value is that it represents all patients operated upon. The richness and the volume of these data would enable doctors to provide their patients with information about outcomes specific to their cancer type, TNM stage, and surgical treatment.

A valuable place for registries is to monitor practice and its variation; ${ }^{10}{ }^{11}$ the institutional pneumonectomy rate in Norway varies from $45 \%$ to $9.5 \%$ of lung resections. The overall resection rate of $16.4 \%$ reported is much higher than current UK practice but may be lower than USA rates. Recent data in Thorax illustrated wide international variation in the resection rate from $7 \%$ in Teesside, UK to $24 \%$ in Varese, Italy. ${ }^{11}$

The hot topic of surgeon and institutional volume versus outcome can also be explored. In this report, as in our own analysis of UK data, ${ }^{12}$ there does not appear to be a "volume effect" with respect to lobectomy, at least not in the narrow terms of perioperative mortality. We concluded that this is perhaps because the surgeons who do few lobectomies nonetheless work within cardiothoracic units and frequently perform complex procedures in the chest. That said, the Norwegian data contain operations performed by general surgeons as well as thoracic surgeons.

However, there are dangers in drawing inferences from differences in outcome between distinct groups. For example, patients who had an upper lobectomy fared better than those who had a lower lobectomy. While this information may be of use in advising patients, it is not a choice available to either patient or surgeon-it is mandated by the site of the cancer. The stress placed on the observation that there was a poorer outcome after pneumonectomy is perhaps inappropriate for similar reasons. This may be because pneumonectomy patients have more extensive cancers, additional morbidity associated with the complications of the pneumonectomy space, or worse survival with less lung parenchyma. The research question would be: for patients in whom both lobectomy and pneumonectomy are options, which operation is associated with a better outcome? We cannot answer this from observational data. Likewise, to perform a sublobar resection is a surgical choice, most likely to be done in patients with co-morbidity. As far as this clinical decision is concerned, it is one of the very few circumstances for which we have randomised trial data ${ }^{13}$ which show that the quicker and simpler sublobar resection is performed at a price; there is more local recurrence and reduced survival. The observational data are in line with this conclusion, but one cannot infer that sublobar resection is never the correct option; indeed, for many patients it may be the only viable surgical option. Strand et al set out the hazard ratios associated with cancer and patient specific explanatory variables (histological type, tumour size, pTNM) alongside factors relating to clinical decisions (table 2), making no distinction between them. To reduce the potential for inappropriate inference, we would favour separate presentation of such data.

That is not to diminish the importance of these observational data, but merely to set limits on their interpretation. Turning to broader issues, a central belief in the treatment of lung cancer, restated in this paper, is that surgical resection has been and remains the leading prospect of cure. We should remind ourselves that this belief (which we share) has never been formally tested. A randomised controlled trial reported in $1963^{14}$ which compared surgery with radiotherapy showed surgery to be associated with longer survival, but that result includes the possibility that radiotherapy causes net harm. The current widely held beliefthat surgery can cure some people with lung cancer-relies on observational case series. For example, it relies on our belief that patients with the most favourable cancers-that is pStage Ia disease-would not have survived untreated at a rate of nearly $50 \%$ at 10 years. For a number of reasons, we have no way of knowing.

The lung cancer epidemic will recede in due course as smoking goes out of fashion and habits change, as they surely must (although in 1919 people said the same of war). Meanwhile, discovering the disease earlier in its course, if that can be achieved, provides a hope of better overall survival figures. But this will not make much difference for cancers where the biological characteristics include a high potential for 
dissemination and the disease is systemic before it can be detected. The prospect of better survival for the many patients staged as inoperable at presentation will not rely on better operations. There is no reason to think that we can clear cancer better than our predecessors. Strand et al ${ }^{4}$ comment that recent improvements in surgical outcomes have been sparse. It is almost certain that the overall improvement in 5 year survival from $26 \%{ }^{15}$ to the $41 \%$ shown in this series is a result of turning down patients for surgical resection by ever more careful preoperative staging. We are likely to resort to combination therapies with surgery being employed alongside present or as yet undefined non-surgical treatments. Given that the envisaged incremental benefits are modest, these combinations are likely to be evaluated in randomised trials. It is likely that there will be a differential degree of benefit depending on the stage of the cancer and the fitness of the patient, but not every scenario is amenable to a trial. Extension of knowledge concerning trial populations to different patient groups will rely on modelling, ${ }^{16}{ }^{17}$ and those models will be based on high quality observational data such as in this Norwegian population based study.

Perhaps because of the belief that surgery can cure some patients with lung cancer, operations for lung cancer are only performed with the intent of cure. However, cure in absolute terms may not be in our gift for many lung cancers. For many diseases (including hypertension, atherosclerosis, and diabetes) we have to satisfy ourselves with control of the manifestations. In breast cancer the view held about 40 years ago that cure could be attained if only surgery was more radical has been rejected. The language is now of remission, whereas in lung cancer we still talk in terms of a crude dichotomy-curative versus palliative intent. The fact is that many patients go on to die of the cancer after treatments given with "curative intent". Surgery may expand its role if it is proved to be a beneficial part of multimodality treatment aimed at modifying the course of disease towards longer and/or better quality survival. Extensions of the role of surgery will need an evidence base and observational series will surely have their place. ${ }^{2}$ The value of this report from Norway is that it provides "some data on all the patients". These are very valuable data indeed, representing the outcomes of 3211 lung cancer operations.

Thorax 2006;61:649-650.

doi: 10.1136/thx.2006.062505

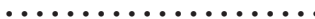

Authors' affiliations

T Treasure, Thoracic Unit, Guy's Hospital, London, UK

M Utley, Clinical Operational Research Unit, University College London, London, UK

Correspondence to: Professor T Treasure,

Thoracic Unit, Guy's Hospital, London SE1 9RT,

UK; tom.treasure@ukgateway.net

Competing interests: none declared.

\section{REFERENCES}

1 Treasure T. A brief history of evidence. In: Treasure T, et al. The evidence for cardiothoracic surgery. Shrewsbury, UK: ffm Publishing Limited, 2005:5-16

2 Rothwell PM. Medical academia is failing patients and clinicians. BMJ 2006:332:863-4.

3 Silvestri GA, Peake $M$, Waller D, et al. A tale of two cities: comparing lung cancer outcomes in
Teesside, UK and Varese, Italy. Thorax 2006:61:188.

4 Strand T-E, Rostad H, Moller B, et al. Survival after resection for primary lung cancer: a population based study of 3211 resected patients. Thorax 2006;61:710-15

5 Bobak M, Kristenson M, Pikhart H, et al. Life span and disability: a cross sectional comparison of Russian and Swedish community based data. BMJ 2004;329:767.

6 Berry RJ, Kihlberg R, Devine O. Impact of misclassification of in vitro fertilisation in studies of folic acid and twinning: modelling using population based Swedish vital records. BMJ 2005;330:815.

7 Hall P, Adami HO, Trichopoulos D, et al. Effect of low doses of ionising radiation in infancy on cognitive function in adulthood: Swedish population based cohort study. BMJ 2004;328: 19.

8 Eaton W, Mortensen PB, Agerbo E, et al. Coeliac disease and schizophrenia: population based case control study with linkage of Danish national registers. BMJ 2004;328:438-9.

9 Hemingway $H$, McCallum A, Shipley $M$, et al. Incidence and prognostic implications of stable angina pectoris among women and men. JAMA 2006;295:1404-11.

10 Sedrakyan A, Van Der MJ, Lewsey J, et al. Variation in use of video assisted thoracic surgery in the United Kingdom. BMJ 2004:329:1011-2.

11 Imperatori A, Harrison RN, Leitch DN, et al. Lung cancer in Teesside (UK) and Varese (Italy): a comparison of management and survival. Thorax 2006;61:232-9.

12 Treasure T, Utley M, Bailey A. Assessment of whether in-hospital mortality for lobectomy is a useful standard for the quality of lung cancer surgery: retrospective study. BMJ 2003;327:73.

13 Ginsberg RJ, Rubinstein LV. Randomized trial of lobectomy versus limited resection for T1 NO nonsmall cell lung cancer. Lung Cancer Study Group. Ann Thorac Surg 1995;60:615-22.

14 Morrison R, Deeley T, Cleland W. The treatment of carcinoma of the bronchus: a clinical trial to compare surgery and supervoltage radiotherapy. Lancet 1963;i:683-4.

15 Belcher JR. Thirty years of surgery for carcinoma of the bronchus. Thorax 1983;38:428-32.

16 Birim O, Kappetein AP, van Klaveren RJ, et al Prognostic factors in non-small cell lung cancer surgery. Eur J Surg Oncol 2006;32:12-23.

17 Utley M, Paschalides C, Treasure T. Informing decisions concerning adjuvant chemotherapy following surgical resection of non-small cell lung cancer: a mathematical modelling study. Lung Cancer 2006 (in press). 